\title{
Acknowledgement of reviewers for 2019
}

\section{Clint N. Dawson ${ }^{1} \cdot$ Mary F. Wheeler ${ }^{1} \cdot$ Ivan $_{\text {Yotov }}{ }^{2}$}

Published online: 15 February 2020

(C) Springer Nature Switzerland AG 2020

The editors and publisher offer their sincere thanks and gratitude to all scientists and experts listed below for devoting their time and effort to review papers during 2019.

Sigurd Ivar Aanonsen
Mohammad Afshari Moein
Khansaa Ahmed
Zhi Yong Ai
Boujemaa Ait-El-Fquih
Denis Allard
Tameem Almani
Faruk Alpak
Richard Amos
Cheng An
Odd Andersen
Pål Andersen
Mauricio Araya
Todd Arbogast
Mark Asch
Christopher Aselmann-Lemon
Masoud Babaei
Hexiang Bai
Raj Banerjee
Markus Bause
Lauren Beckingham
Masoud Behzadinasab
James Bennett
Haluk Beyenal
J. Eric Bickel
Radim Blaheta
Christian Boehm

Edo Boek
Jeff Boisvert
Manuel Borregales
Villiam Bortolotti
Claude Boutin
Zyed Bouzarkouna
Frode Bratvedt
Kyrre Bratvedt
Carina Bringedal
Jérémie Bruyelle
Vladislav Bukshtynov
Daniel Busby
Troy Butler
Jef Caers
Nicola Castelletto
Romain Chassagne
Kuldeep Chaudhary
Théophile Chaumont-Frelet
Ann Chen
Bailian Chen
Bin Chen
Chaohui Chen
Huangxin Chen
Yujie Chen
Jinkun Cheng
Jinhyun Choo
Eric Chung

\author{
Holger Class \\ François Clayer \\ Emmanuel Cocher \\ Andres Codas \\ Alberto Cominelli \\ Jose Victor Contreras Sandia \\ Matthias Cremon \\ Saumik Dana \\ Jonas De Basabe \\ Laurent De Windt \\ Ernesto Della Rossa \\ Mojdeh Delshad \\ Vasily Demyanov \\ Lichi Deng \\ Flavio Dickstein \\ Shuaiwei Ding \\ Didier-Yu Ding \\ Vladimir Druskin \\ Svetlana Dubinkina \\ Lou Durlofsky \\ David Edwards \\ Michael Edwards \\ Silvia Ehrmann \\ Mohamed El-Amin \\ Ahmed ElSheikh \\ Alexandre Emerick \\ Jocelyne Erhel
}

Clint N. Dawson

clint@ices.utexas.edu

1 Department of Mechanical and Industrial Engineering, University of Texas at Austin, Austin, TX, USA

2 Department of Mechanical and Industrial Engineering, University of Pittsburgh, Pittsburgh, PA, USA 
Samuel Estes

Geir Evensen

Robert Eymard

Isabelle Faille

Xiaolin Fan

Arash Fathi

Qihong Feng

Runhai Feng

Yin Feng

Massimiliano Ferronato

Bernd Flemisch

Horacio Florez

Rahul-Mark Fonseca

Luca Formaggia

Fahim Forouzanfar

Kristian Fossum

Andrea Franceschini

Pengcheng $\mathrm{Fu}$

Radek Fucik

Gorgonio Fuentes

Alessio Fumagalli

Ilya Fursov

Quan Gan

Guohua Gao

Sarah Gasda

Francisco Gaspar

Saba Gharehdash

Richard Gibson

Marie Ann Giddins

Thomas Gimmi

David Ginsbourger

Ingeborg Gjerde

Takashi Goda

Sophie Godefroy

Sonia Gomes

Jaime Gomez-Hernandez

Jean Gossuin

Dario Grana

Christopher Green

Janek Greskowiak

Sebastian Gries

Dominique Guerillot

Dayal Gunasekera

Zhenyu Guo

Maryam Hadavand

Morteza Haghighat Sefat

Hadi Hajibeygi

Hamidreza Hamdi

Francois Hamon

Dongxu Han

Jincong $\mathrm{He}$

Mohammad Reza Heidari

Rainer Helmig
Thomas Hermans

Julio Hoffimann

Bernardo Horowitz

Hussein Hoteit

Xiaozhe $\mathrm{Hu}$

Marco Iglesias

Oleg Iliev

Ilya Indrupskiy

Olaf Ippisch

Joel Ita

Behnam Jafarpour

Atefeh Jahandideh

Etelvina Javierre

Eleanor Jenkins

Hoonyoung Jeong

Rafael Jesus de Moraes

Su Jiang

Wencheng Jin

Tom Jonsthovel

Gerard Joosten

Peter Kang

Eirik Keilegavlen

W. Kennedy

Michel Kern

Akhtar Khan

Eldar Khattatov

Amin Kiaghadi

John Killough

Michael King

Ole Klein

Øystein Klemetsdal

Denis Klemin

Hector Klie

Robert Klöfkorn

Peter Knabner

Uwe Köcher

Amalia Kokkinaki

Dmitry Koroteev

Antonina Kozlova

Paulina Krakowska

Serge Kräutle

Stein Krogstad

Shyamini Kularathna

Dhananjay Kumar

Kundan Kumar

Rajiv Kumar

Stelios Kyriacou

Eric Laloy

Duc Le

Sanghyun Lee

Seong Lee

Young Ju Lee

Olwijn Leeuwenburgh
Qinghua Lei

Jeremy Levesley

Jingfa Li

Jingzhe Li

Lewis Li

Yang Li

Qinzhuo Liao

Knut-Andreas Lie

Jeen-Shang Lin

Min Liu

Piyang Liu

Ruijie Liu

Rolf Lorentzen

Abimael Loula

Xiaodong Luo

Thang Luong

Maria Lymbery

Jianjun Ma

Wei Ma

Brad Mallison

Denis Marcotte

Kent-Andre Mardal

Chris Marone

Diego Mas Ivars

Roland Masson

Yder Masson

Sebastien Matringe

Steven Mattis

Pawel Matuszyk

Ulrich Mayer

Mark McClure

Yashar Mehmani

Riccardo Mel

Andro Mikelic

Baehyun Min

Susan Minkoff

Siddharth Misra

Koondanibha Mitra

Arthur Moncorge

Olav Møyner

Tapan Mukerji

Marcio Murad

Trine Mykkeltvedt

Arne Naegel

Geir Naevdal

Jostein Natvig

Erik Nesvold

Crystal Ng

Hans Ngodock

Youjun Ning

Behzad Nobakht

Benoit Noetinger

Jan Nordbotten 
Jonathan Norris

Sergey Oladyshkin

Feyisayo Olalotiti-Lawal

Diego Oliveira

Dean Oliver

Jerome Onwunalu

Wenxiao Pan

Nishant Panda

Umberto Perego

Mike Pereira

Felipe Pereira

Malgorzata Peszynska

Elisabeth Peters

Øystein Pettersen

Rachares Petvipusit

Geraldine Pichot

Guillaume Pirot

Phillippe Poncet

Iuliu Sorin Pop

Dimitrii Posvyanskii

Babak Poursartip

Anshuman Pradhan

M. K. Priyan

Patrick Raanes

Florin Radu

Javad Rafiee

Kashif Rashid

Xavier Raynaud

Don Resio

Albert C. Reynolds

Calogero Benedetto Rizzo

Adolfo Rodriguez

Pamphile T. Roy

Gianluigi Rozza

Juan Ruiz

Iryna Rybak

George Saad

Amgad Salama

Pierre Samier

Luiz Sampaio

Boris Samson

Omer San

Pallav Sarma

Ralf Schulze-Riegert

Anna Scotti
Bogdan Sebacher

Mohammad Sedaghat

Mrinal Sen

Marjan Sherafati

Soham Sheth

Alireza Rangriz Shokri

Ralph Showalter

Khemraj Shukla

Majid Siavashi

Gurpreet Singh

Arne Skorstad

Jeroen Snippe

Pierre Sochala

Kaveh sookhaklari

Helio Souto

Eduardo Souza

Carl Steefel

Karl Stephen

Terry Stone

Andreas Stordal

Jonathan Stroud

Michael Suess

Wenyue Sun

Yunwei Sun

Erick Talarico

Daniel Tartakovsky

Soren Taverniers

Aretha Teckentrup

Matei Tene

Emmanuel Tertre

Pavel Tomin

Christophe Tournassat

Philippe Ungerer

Ciriello Valentina

Al Valocchi

Jan Vanderborght

J.A. Vargas-Guzman

Eric Verschuur

Giulio Vignoli

Jeroen Vink

Hai Vo

Oleg Volkov

Denis Voskov

Femke Vossepoel

Siddhant Wahal
Daobing Wang

Enjiang Wang

Jiehao Wang

Min Wang

Peng Wang

Yi Wang

Christoph Wanner

Xian-Huan Wen

Holger Wendland

Dieter Werthmuller

Laurent White

Thomas Wick

Christian Wieners

Tim Wildey

Christian Wolfsteiner

Langping Wu

Chiyu Xie

Yan Xu

Bicheng Yan

Changdong Yang

Jianhui Yang

Pengliang Yang

Yannis Yortsos

Ángel Yustres

Hardikkumar Zalavadia

Fabiana Zama

Jessica Zhang

Tuanfeng Zhang

Xiangliang Zhang

Yanbin Zhang

Yong Zhao

Polina Zheglova

Hong Zheng

Liange Zheng

He Zhong

Yifan Zhou

Hejun Zhu

Wancheng Zhu

Murtaza Ziauddin

Ludmil Zikatanov

Robert Zimmerman

Liangchao Zou

Paolo Zunino

Chen Zuo

We apologize for any errors or inadvertent omissions. 